\title{
Magnitude of Presumptive Tuberculosis Cases, in the Rural Communities of North Western and North Eastern Parts of Amhara Regional State, Ethiopia
}

Araya Mengistu $^{1^{*}}$, Seleshe Nigatu ${ }^{1}$, Tadesse Guadu ${ }^{2}$, Elias Kebede ${ }^{1}$, Bimrew Admasu ${ }^{1}$, Basaznew Bogale ${ }^{1}$, Atnaf Alebe $^{1}$, Samuel Sebsbie ${ }^{3}$, Adugna Burju $^{4}$, Wendweson Kumlachew ${ }^{5}$, Mezgebu Asmiro ${ }^{6}$, and Wubegzier Mekonnen ${ }^{7}$

${ }^{1}$ Faculty of Veterinary Medicine, University of Gondar, Ethiopia

${ }^{2}$ College of Medicine and Health Sciences, Institute of Public Health, University of Gondar, Ethiopia

${ }^{3}$ School of Economics, University of Gondar, Ethiopia

${ }^{4}$ North Gondar Agricultural Department, Ethiopia

${ }^{5}$ Kombolcha Regional Veterinary Laboratory, Ethiopia

${ }^{6}$ North Wollo Agricultural Department, Ethiopia

${ }^{7}$ School of Public Health, Addis Ababa University, Ethiopia

*Corresponding author: Dr. Araya Mengistu, Faculty of Veterinary Medicine, University of Gondar, Ethiopia, E-mail: armen.kassa@gmail.com Received date: Aug 2, 2016; Accepted date: Sep 27, 2016; Published date: Oct 7, 2016

Copyright: $\odot 2016$ Mengistu A, et al. This is an open-access article distributed under the terms of the Creative Commons Attribution License, which permits unrestricted use, distribution, and reproduction in any medium, provided the original author and source are credited.

\begin{abstract}
Despite the availability of highly efficacious treatment for decades, TB remains a major global public health problem. Nearly one third of the world's population is infected with Mycobacterium tuberculosis (MTB) and hence at risk of developing active disease. Tuberculosis is a major cause of morbidity and mortality in Ethiopia, and the country belongs to one of the most affected high burden countries in the world. Presumptive tuberculosis cases which are individuals having cough duration of more than two weeks has been used as a quick TB diagnosis in rural settings. Determining the prevalence of presumptive tuberculosis cases in selected rural communities of north western and north eastern parts of Amhara regional state was the study objective. For this study 10 districts from North Gondar and North Wollo zones were purposefully selected and 26 kebeles were included where Kebeles selected randomly based on the district tuberculosis report. The study was cross-sectional conducted from March 15, 2016 to March 30, 2016. All households in the selected kebeles were considered as a study population. A house to house check was also used as message dissemination to all dwellers in the kebele to register presumptive tuberculosis cases and all reported to have the problem were registered. A pretested semi-structured questionnaire was administered to collect socio demographic and related information. The questionnaires were filled by data collectors and all the responses were kept confidential. The overall prevalence of presumptive tuberculosis at household level in both zones was $2 \%$. A relatively higher household prevalence were found on Gebsye, Achera, Dib-bahir, Dabat Zuria, Kino, and Debot kebeles' with 3.9\%, 4.0\%, 5.3\%, 6.0\%, 7.0\% and 8.1\%, respectively. The mean presumptive cases per kebele was 28 cases. Dibbahir (57 cases), Achera (64 cases) and Debot (102 cases) kebeles' were found to have the highest count, which were more than twice the mean number of cases on the studied kebeles. The mean proportion of presumptive tuberculosis cases was found to be 441 cases per 100,000 population and the highest rates were found on Dabat Zuria, Achera, Dib-bahir, Kino and Debot kebeles' with 912 cases, 939 cases, 1230 cases, 1466 cases and 1888 cases, respectively per 100,000 populations. The magnitude of presumptive tuberculosis cases in the study areas was relatively high. Intensive health education, early case detection, frequent surveillance, diagnosis, real causes of chronic cough identifications \& treatment are suggested to reduce the probable spread of the tuberculosis to a wider population.
\end{abstract}

Keywords: Tuberculosis; Presumptive cases; Housebound; North East; North West; Ethiopia

\section{Background}

TB is a chronic mycobacterial contagious disease caused primarily by $M$. tuberculosis, and occasionally by $M$. bovis and $M$. africanum in humans. The disease is, spreading through the air by coughing, sneezing, or spitting $[1,2]$ and it has remained a public health challenge in the world and considered as a major cause of morbidity and mortality. Worldwide there are about 2 billion persons infected with TB, of which $5-10 \%$ will become sick in their life time [3,4]. Human tuberculosis is known in Ethiopia; according to the latest estimate, Ethiopia is one of the highest tuberculosis burden countries in the world, which ranks 8th amongst the 22 global tuberculosis high- burden countries. In a study carried out in 2014 in the country an overall prevalence of MDR-TB $5.7 \%$ was reported where it was $2.3 \%$ and $13.9 \%$ among new cases and previously treated cases, respectively [5].

The most common source of infection for TB is the human case whose sputum is positive for the tubercle bacilli (active TB patient). Moreover, those who are totally not treated or treated inadequately can also be used as a source of infection. People who have just started on TB treatment and who has a poor response to treatment can also be used as a source of infection and milk containing Mycobacterium bovis could be a source of infection to humans $[1,6-8]$. The source of infection for tuberculosis is mainly those patients who harbour the infectious agents; however, it has also been reported in a wide range of domestic and wild animals most frequently in animals, which have close and prolonged contact with humans. Among domestic animals, 
Citation: Mengistu A, Nigatu S, Guadu T, Kebede E, Admasu B, et al. (2016) Magnitude of Presumptive Tuberculosis Cases, in the Rural Communities of North Western and North Eastern Parts of Amhara Regional State, Ethiopia. Clin Microbiol 5: 261. doi: $10.4172 / 2327-5073.1000261$

Page 2 of 4

infection with $M$. tuberculosis was identified in cattle and isolated from pathological lesions and milk of intradermal skin tested positive animals. The prevalence of diagnosis infection in cattle herds could reach to $7.4 \%$ [9]. It has also been reported that animal attendants with active pulmonary tuberculosis can serve as a source of $M$. tuberculosis for animals. Conditions like sharing common houses will facilitate infection of both populations [10].

According to WHO those individuals who suffered from cough with a duration of more than two weeks are expected to visit health institutes for tuberculosis diagnosis [11]. In most of our societies, chronic cough in a family is not seen as a problem. In most of our community health seeking behavior is less compared to others. Delay in the diagnosis of the problem might lead to severe health consequences apart from the possibility of being active pulmonary TB. Individuals who suffered from chronic cough will not devote much of their time to work and at times wasting time in finding solutions and therefore, resulting in lower participations in agricultural activities. Apart from the societal impacts of chronic cough, the indirect and direct economic losses could be magnificent if left untreated. The objective of this study was to assess the magnitude of presumptive tuberculosis cases in selected districts of North Gondar and North Wollo zones.

\section{Material and Methods}

\section{Study areas}

The study was conducted in 10 districts of North Gondar and North Wollo zones of the Amahra Regional State. North Gonadr zone is situated at Latitude: $12^{\circ} 39^{\prime} 29.05^{\prime \prime}$ and Longitude: $37^{\circ} 5^{\prime} 18.14$. It is located at a distant of about $710 \mathrm{~km}$ from Addis Ababa, capital of Ethiopia. While North Wollo zone is situated at Latitude: $11^{\circ} 55^{\prime} 12^{\prime \prime \prime \prime}$ and Longitude: $39^{\circ} 5^{\prime} 59.99^{\prime \prime}$ and it is located at a distant of about 520 $\mathrm{km}$ from Addis Ababa, capital of Ethiopia. Based on the 2007 Census conducted by the Central Statistical Agency of Ethiopia (CSA), north Gondar zone has a total population of 2,929,628, of whom 1,486,040 were men and 1,443,588 women with a population density of 63.76 and more than $74 \%$ lives in rural areas. A total of 654,803 households were counted in this Zone, which results in an average of 4.47 persons to a household. North wollo Zone has a total population of 1, 500, 303, of whom 752,895 are men and 747,408 women with a population density of 123.25 . More than $87 \%$ of them are living in the rural settings. A total of 355,974 households were counted in this Zone, which results in an average of 4.21 persons to households [12]. A study conducted to evaluate health seeking behaviors in North Wollo zone indicated that $62.3 \%$ of the interviewed TB patients responded that they visit health institutes for consultation after 30 days of onset of the clinical signs [13]. Six and 4 districts were selected from North Gondar and North Wollo zones, respectively. Two to three Kebeles were included from each district.

\section{Study design and Selection}

The study was a cross-sectional one conducted from March 15, 2016 to March 30, 2016. The study zones and districts were selected purposefully and based on some previous experiences. Study kebeles were selected based on relatively high tuberculosis cases or suspected case reports. If the numbers are more than three kebeles per district we used a simple random sampling technique to select the Kebeles. The health extension workers in each kebele tour house to house and enquire the presence of persons having a chronic cough with a duration of more than two weeks [11].

\section{Source and study Populations}

Usual residents of the two study zones are considered as the source population while households in selected districts are considered as the study population.

\section{Exclusion criteria}

For this study those under TB or other treatment against cough were not included in the study.

\section{Data collection}

A pretested structured and semi-structured questionnaire was administered. All households were taken as a cluster and in each kebele, the required data were collected by using a house-to-house move and dissemination of the information through the developmental or health army teams. Those who had cough more than 2 weeks were interviewed on the spot and those heard the message were appear to the health post and gave information. The questionnaires were filled by data collectors.

\section{Data management and analysis}

The collected questionnaire was checked for its completeness. The variables were coded as $0(\mathrm{No})$ and 1 (Yes) and data were entered into excel spread sheet by the data clerk. The data were edited and cleared before analysis and exported to SPSS (20.0) Statistical Packages for Social Sciences [14]. Purely descriptive statistics was used to analyze the data and table and graphs as well as charts were used to present the findings.

\section{Ethical consideration}

The research was approved by the university of Gondar ethical review board and then Amhara National health research center. Before starting the research permission was obtained from each zonal health departments and then district health offices. Almost all activities were facilitated by district tuberculosis officers. All participants gave oral consents and were explained about the study and told to withdraw from the research, if they are not willing. Those who identified as presumptive tuberculosis cases were registered by health extension workers and subjected to acid fast bacilli stain for further diagnosis and actions and these mandates was given to health extension workers to follow all the forthcoming procedures.

\section{Results}

Of the participants $53.2 \%, 88.3 \%, 46.4 \%$ and $93.0 \%$ where males, farmers, illiterate and orthodox Christianity followers respectively (Table 1).

Six districts were included from North Gondar Zone accounting about $60 \%$ of the area and $461(68 \%)$ of presumptive Tuberculosis cases were from it. Highest presumptive TB cases were recorded in Dabat (20\%) (N. Gondar) and Gubalafto (20.3\%) (N. Wollo) districts (Figure 1). The overall prevalence of presumptive tuberculosis cases at household level was $2 \%$. A relatively higher household prevalence were found on Gebsye, Achera, Dib-bahir, Dabat Zuria, Kino, and Debot kebeles' with $3.9 \%, 4.0 \%, 5.3 \%, 6.0 \%, 7.0 \%$ and $8.1 \%$, respectively. The 
Citation: Mengistu A, Nigatu S, Guadu T, Kebede E, Admasu B, et al. (2016) Magnitude of Presumptive Tuberculosis Cases, in the Rural Communities of North Western and North Eastern Parts of Amhara Regional State, Ethiopia. Clin Microbiol 5: 261. doi: $10.4172 / 2327-5073.1000261$

Page 3 of 4

mean presumptive cases per kebele was $28+15.6$ (95\% CI: 26.84-29.16) cases. Dibbahir (57 cases), Achera (64 cases) and Debot (102 cases) kebeles' were found to have the highest count, which were more than twice the mean number of cases on the studied kebeles. The mean proportion of presumptive tuberculosis cases was found to be 441 cases per 100,000 population and the highest rates were found on Dabat Zuria, Achera, Dib-bahir, Kino and Debot kebeles' with 912 cases, 939 cases, 1230 cases, 1466 cases and 1888 cases, respectively per 100, 000 populations.

\begin{tabular}{|c|c|c|c|c|c|}
\hline Descriptive & Frequency & Per cent & Descriptive & Frequency & Per cent \\
\hline Sex & & & Religion & & \\
\hline Male & 362 & 53.2 & Orthodox Christian & 633 & 93 \\
\hline Female & 319 & 46.8 & Muslim & 48 & 7 \\
\hline Occupation and income & & & Education & & \\
\hline Farmer & 601 & 88.3 & Illiterate & 316 & 46.4 \\
\hline Employee & 20 & 3 & Primary & 214 & 31.4 \\
\hline Trade & 7 & 1 & Secondary & 30 & 4.4 \\
\hline \multirow[t]{2}{*}{ Other } & 53 & 7.7 & College/University & 9 & 1.3 \\
\hline & & & Traditional & 112 & 16.4 \\
\hline
\end{tabular}

Table 1: Sociodemographic characteristics of presumptive TB cases in the study areas, 2016.

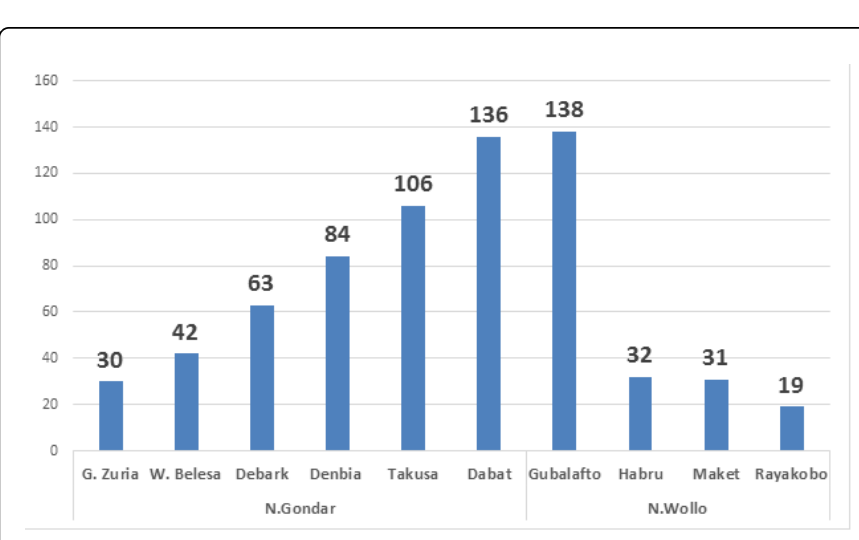

Figure 1: Distribution of Presumptive TB cases by Districts, 2016.

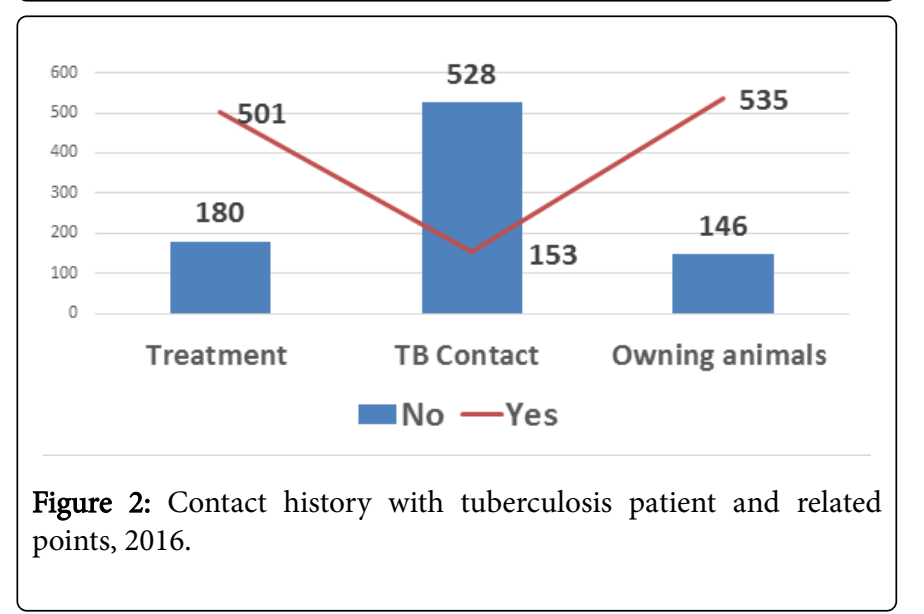

Of the participants 501 (73.6\%), 535 (78.6\%) and 153 (22.3\%) tried to take treatments (either modern or traditional), owned animals and had contact with confirmed tuberculosis patients, respectively (Figure 2).

\section{Discussion}

According to WHO 2014 global report of TB, the estimated prevalence of $\mathrm{TB}$ in Ethiopia was 211 per 100,000 populations $(0.211 \%)$ [15]. In the present study, the overall prevalence of presumptive TB cases was $2 \%$, which is higher than the above figure. The difference could be due to the fact that our study participants were TB suspected cases within the selected kebeles and the methodology used compared to WHO report.

On the other hand, a study conducted in Bahir Dar health center, Northwest Ethiopia among presumptive pulmonary TB patients who visited the health center the overall prevalence of smear positive PTB was 6.8\% [16]. On similar studies conducted in South and Eastern part of Ethiopia, among presumptive cases the prevalence of smear positive TB was $9.2 \%$ [17] and $21.6 \%$ [5], respectively which are significantly higher than the finding of the present study. The difference could be due to the fact that our data was collected based on self-report and population difference.

The mean proportion of presumptive tuberculosis cases was found to be 441 cases per 100,000 populations, whereas the WHO annual estimate (2014) of an overall TB prevalence of the country is 211 per 100000 populations [15]. The higher prevalence in the current study could be due to the methodology used, study population, study setting as well as study time difference.

Tuberculosis affects all age and sex groups. According to a study in Ethiopia the age groups 15-45 were more affected than other groups [5]. In contrast, in the present study relatively high prevalence of presumptive TB (11\%) was observed among people aged between 45-50 groups followed by 35-40 and 25-30 age groups with 10.7 and $10.1 \%$ prevalence respectively. On the other hand, relatively smaller prevalence $(4.1 \%)$ were found on study subjects belonging 15-20 age 
Citation: Mengistu A, Nigatu S, Guadu T, Kebede E, Admasu B, et al. (2016) Magnitude of Presumptive Tuberculosis Cases, in the Rural Communities of North Western and North Eastern Parts of Amhara Regional State, Ethiopia. Clin Microbiol 5: 261. doi: $10.4172 / 2327-5073.1000261$

Page 4 of 4

groups, while the mean age of the study participants were 28.4 years. This can be explained by the fact that our study participants who were of course presumptive TB suspected cases in which the possibility of being positive for TB is higher as age of study subjects increases.

According to 2014 World TB report, WHO declared that most TB cases and deaths occur among males, which is in line with the present study where $53.2 \%$ of the presumptive TB cases were males. However burden of the disease is also high among women. The global attention to maternal and child health created demand for interest in detection and management of TB among women [15].

In relation to contact history, more than three fourth (77.5\%) of the respondents were associated with a history of having contact with TB patients. This result was a little bit higher than Yohanes et al. in 2012 [18] where $41 \%$ of the study participants in metehara sugar factory hospital suspected cases had contact with chronic coughers (TB infected patients). On the other hand, a lower close contact history (only 4.5\%) was also found on the first Ethiopian national population based tuberculosis prevalence survey in 2011 [19]. The difference could due to the difference in diagnostic approach, population difference and study time.

In this study, the majority of presumptive $\mathrm{TB}$ cases (88.3\%) were farmers by profession and illiterates (46.4\%) regarding their educational background. Similarly, farmers account about $49.6 \%$ on the first Ethiopian national population based tuberculosis prevalence survey in 2011 [19]. This could be attributed by the inclusion of large sample size and study approaches.

As far as family size is concerned, household having 5 family size was found to have greater proportion of presumptive TB cases. This is in line with similar study conducted in Bahir Dar health center, Northwest Ethiopia [16]. This could explained by the sociodemographic similarity of the study subjects and closeness of the study period. Considering only the prevalence of presumptive tuberculosis cases, lack of collecting sputum samples, lack of identification of the possible causes of chronic coughers were the limitations of this study.

\section{Conclusion and Recommendations}

In conclusion, the prevalence of presumptive $\mathrm{TB}$ cases at household level was $2 \%$ in the rural communities of north western and north eastern parts of Amhara regional state. More frequencies regarding presumptive TB cases were reported among 45-50 age groups, male sex, and households having 5 family sizes. Hence, it is recommend strict presumptive TB screening, early case finding and treatment among households and intensive health education to reduce the chance of spread of the disease. It is also wise to recommend longitudinal studies with advanced laboratory techniques and chronic cough causes identifications.

\section{Acknowledgement}

The team would like to extend many thanks to University of Gondar, V/president office for research and community services, faculty of veterinary medicine and those communities in the selected kebeles as well as health extension works along with respective tuberculosis officers.

\section{References}

1. Hinshaw W (1933) Tuberculosis of human origin in an Amazon parrot. Am Rev Tuberc 28: 273-8.

2. Hershberg R, Lipatov M, Small PM, Sheffer H, Niemann S, et al. (2008) High functional diversity in Mycobacterium tuberculosis driven by genetic drift and human demography. PLoS Biol 6: e311.

3. Shapira Y, Agmon-Levin N, Shoenfeld Y (2010) Mycobacterium tuberculosis, autoimmunity, and vitamin D. Center for Autoimmune Diseases, Chaim Sheba Medical Center, Tel-Hashomer 52621, Israel.

4. Global Tuberculosis report. 2005.

5. Mekonnen F, Tessema B, Moges F, Gelaw A, Eshetie S, et al. (2015) Multidrug resistant tuberculosis: prevalence and risk factors in districts of metema and west armachiho, Northwest Ethiopia. BMC Infect Dis 15: 461.

6. Woerper M, Rosskoph W (1983) Proceedings of the Annual Meeting of the Association of Avian Veterinarians. Retro-orbital Mycobacterium tuberculosis infection in a yellow-naped Amazon parrot (Amazona ochrocephala auropalliata). pp. 71-6.

7. Smith NH, Gordon SV, de la Rua-Domenech R, Clifton-Hadley RS, Hewinson RG (2006) Bottlenecks and broomsticks: the molecular evolution of Mycobacterium bovis. Nat Rev Microbiol 4: 670-681.

8. Ocepek M, Pate M, Zolnir-Dovc M, Poljak M (2005) Transmission of Mycobacterium tuberculosis from human to cattle. J Clin Microbiol 43: 3555-3557.

9. Smith IGN (1984) A herd breakdown due to Mycobacterium tuberculosis. State Vet J 38: 40-44.

10. Wirth T, Hildebrand F, Allix-Béguec C, Wölbeling F, Kubica T, et al. (2008) Origin, spread and demography of the Mycobacterium tuberculosis complex. PLoS Pathog 4: e1000160.

11. Central Statistics Agency, Ethiopia; A Population Census. 2007.

12. Dejen T, Ephrem A, Tesfahun M, Takele T (2016) Delay in Seeking Health Care and associated Factors among Pulmonary Tuberculosis Patients in North Wollo Zone, Northeast Ethiopia. Institution Based Cross-sectional Study. Archives of clinical Microbiology.

13. Statistical Packages for Social Sciences (SPSS).20.

14. Pretorius C, Glaziou P, Dodd PJ, White R, Houben R (2014) Using the TIME model in Spectrum to estimate tuberculosis-HIV incidence and mortality. AIDS 4: S477-487.

15. Derbie A, Mekonnen D (2015) Prevalence of smear positive pulmonary tuberculosis among patients visiting Bahir Dar health center, Northwest Ethiopia. International Journal of Public Health and Epidemiology ISSN 2326-7291 Vol. 4, pp. 179-182.

16. Tulu B, Dida N, Kassa Y, Taye B (2014) Smear positive pulmonary tuberculosis and its risk factors among tuberculosis suspect in South East Ethiopia; a hospital based cross-sectional study. BMC Res Notes 7: 285

17. Mekonnen A (2014) Smear-positive pulmonary tuberculosis and AFB examination practices according to the standard checklist of WHO's tuberculosis laboratory assessment tool in three governmental hospitals, Eastern Ethiopia. BMC Res Notes 7: 295.

18. Yohanes A, Abera S, Ali S (2012) Smear positive pulmonary tuberculosis among suspected patients attending Metehara sugar factory hospital; eastern Ethiopia. Afr Health Sci12: 325-30.

19. Tuberculosis Prevalence Survey. EFMOH, July 2011. 